\title{
Prospective Study on Waist Circumference and Risk of All-Cause and Cardiovascular Mortality
} - Pooled Analysis of Japanese Community-Based Studies -

\author{
Isao Saito, MD, PhD; Yoshihiro Kokubo, MD, PhD; Yutaka Kiyohara, MD, PhD; \\ Yasufumi Doi, MD, PhD; Shigeyuki Saitoh, MD, PhD; \\ Hirofumi Ohnishi, MD, PhD; Yoshihiro Miyamoto, MD, PhD
}

\begin{abstract}
Background: The aim of the present study was to clarify the association between waist circumference and all-cause and cardiovascular disease (CVD) mortality risk in relatively lean Japanese subjects.

Methods and Results: A total of 3,554 men and 4,472 women who had no history of CVD were examined and their waist circumference measured at baseline. The subjects were aged $\geq 40$ years and were obtained from 3 prospective cohort studies during 1988-1996. Hazard ratios for all-cause and CVD mortality were analyzed over a follow-up period of 14.7 years using a Cox proportional hazards model and penalized spline method, after adjustment for study cohort, age, smoking, alcohol drinking, hypertension, dyslipidemia, and diabetes. Compared with the lowest quintile, the highest quintile of waist circumference in men was associated with a linear reduction in all-cause mortality risk (multivariate-adjusted hazard ratio, 0.73; 95\% confidence interval: 0.60-0.89; P for trend=0.001). CVD mortality risk was increased in men aged $\leq 65$ years with a higher waist circumference. This relationship was $U$-shaped. Waist circumference was not associated with all-cause or CVD mortality risk in women.
\end{abstract}

Conclusions: Waist circumference was associated inversely with increased risk of all-cause death in men, but not in women. Middle-aged men with a greater waist circumference potentially have an increased risk of CVD mortality. (Circ J 2012; 76: 2867-2874)

Key Words: Cardiovascular disease; Mortality; Prospective study; Waist circumference

$\mathbf{R}$ ecently, a prospective study in more than 1 million Asian subjects has confirmed that there is a U-shaped association between body mass index (BMI) and the risk of death from cancer, cardiovascular disease (CVD), and other causes. ${ }^{1}$ But previous epidemiological studies in Japan suggested that increased BMI was unlikely to influence mortality risk, ${ }^{2,3}$ whereas a lower BMI or weight loss markedly raised this risk. ${ }^{4}$

In contrast, it has been reported that Japanese overweight or obese subjects have an increased risk for incident coronary heart disease or ischemic stroke. ${ }^{5,6}$ The Hisayama study in Japan documented that increased BMI was associated with an increased risk of stroke in women, but not in men. ${ }^{7}$ An enhanced risk for incident CVD and stroke associated with increased waist circumference was also found only in women in the Suita study. ${ }^{8}$ Taken together, these findings indicate that overweight or obesity has an impact on incident CVD in Japanese men and women, except for stroke in men.

A large European prospective study documented that abdominal obesity, as indexed by waist circumference, had a Jshaped association with mortality risk. ${ }^{9}$ That study showed that overweight or obesity, assessed by BMI or waist circumference, was associated closely with increased risk of CVD mortality in Caucasian subjects. Compared with indices of adiposity, a waist circumference signifying central obesity was a stronger marker for predicting CVD mortality than BMI. ${ }^{10} \mathrm{~A}$ recent meta-analysis in Caucasian subjects found increased all-cause and CVD mortality risks for elderly people (65-74 years old) with an increased waist circumference across BMI categories and for underweight elderly people. ${ }^{11}$ The association of waist circumference levels with CVD mortality risk, however, remains to be determined in Japanese adults. Therefore, to better

Received November 6, 2011; revised manuscript received June 19, 2012; accepted July 17, 2012; released online August 8, 2012 Time for primary review: 22 days

Department of Public Health, Social Medicine and Medical Informatics, Ehime University Graduate School of Medicine, Toon (I.S.); Department of Preventive Cardiology, National Cerebral and Cardiovascular Center, Suita (Y. Kokubo, Y.M.); Department of Environmental Medicine (Y. Kiyohara), Department of Medicine and Clinical Science (Y.D.), Graduate School of Medical Sciences, Kyushu University, Fukuoka; and Second Department of Internal Medicine, Sapporo Medical University of Medicine, Sapporo (S.S., H.O.), Japan

Mailing address: Isao Saito, MD, PhD, Department of Public Health, Social Medicine and Medical Informatics, Ehime University Graduate School of Medicine, 454 Shitsukawa, Toon 791-0295, Japan. E-mail: saitoi@m.ehime-u.ac.jp

ISSN-1346-9843 doi:10.1253/circj.CJ-11-1259

All rights are reserved to the Japanese Circulation Society. For permissions, please e-mail: cj@j-circ.or.jp 


\begin{tabular}{lccc} 
Table 1. Subject Characteristics & \multicolumn{3}{c}{ Cohorts } \\
\cline { 2 - 4 } & Tanno/Sobetsu & Suita & Hisayama \\
Baseline year & 1994 & $1991-1996$ & 1988 \\
$\mathrm{n}$ & 1,525 & 4,135 & 2,366 \\
Follow-up period (years) & 11.3 & 14.2 & 17.9 \\
No. deaths & 226 & 792 & 693 \\
No. CVD deaths & 71 & 174 & 202 \\
Age (years) & $62.1 \pm 9.7$ & $59.4 \pm 10.9$ & $56.5 \pm 10.4$ \\
Male (\%) & 40.7 & 43.2 & 46.2 \\
WC (cm) & $79.0 \pm 9.6$ & $81.3 \pm 9.1$ & $81.7 \pm 9.2$ \\
BMI (kg/m²) & $23.5 \pm 3.1$ & $22.6 \pm 3.0$ & $23.0 \pm 3.1$ \\
SBP (mmHg) & $136.9 \pm 20.4$ & $126.6 \pm 20.5$ & $132.3 \pm 20.6$ \\
DBP (mmHg) & $79.0 \pm 9.8$ & $76.6 \pm 11.4$ & $77.9 \pm 11.4$ \\
Hypertension (\%) & 47.7 & 31.3 & 40.0 \\
Dyslipidemia (\%) & 41.8 & 54.1 & 60.8 \\
Diabetes (\%) & 6.5 & 4.3 & 9.0 \\
Current smoker (\%) & 25.1 & 26.4 & 25.4 \\
Regular alcohol drinker (\%) & 17.3 & 59.8 & 22.9 \\
\hline
\end{tabular}

Data given as mean $\pm \mathrm{SD}, \mathrm{n}$ or $\%$.

BMI, body mass index; CVD, cardiovascular disease; DBP, diastolic blood pressure; SBP, systolic blood pressure; WC, waist circumference.

understand the association of abdominal obesity with all-cause and CVD mortality risk, we conducted a 14.7-year prospective study in the general Japanese population.

\section{Methods}

\section{Subjects}

The subjects consisted of the participants, aged between 40 and 90 years, of 3 large Japanese epidemiological studies: the Tanno and Sobetsu study $(\mathrm{n}=1,627)$, the Suita study $(\mathrm{n}=4,278)$, and the Hisayama study $(n=2,487)$. We selected 3,554 men and 4,472 women from these studies who had no history of ischemic heart disease (IHD) or stroke and who had their waist circumference measured at baseline.

Briefly, subjects in the Tanno and Sobetsu study were recruited from annual health check-ups carried out in 1994 in the towns of Hokkaido Island, located in the northern part of Japan. ${ }^{12}$ The Suita study, which was performed in an urban area of Japan (Osaka), also enrolled individuals for regular health check-ups between 1991 and $1996 .{ }^{8}$ The Hisayama study, which was conducted in a suburban town located in Kyushu Island in the southern part of Japan, was established in 1961 for all residents $\geq 40$ years of age, and we used the third cohort, which was established in $1988 .{ }^{13}$ Details of recruitment and the procedures for all studies have been described elsewhere..$^{8,12,13}$

\section{Measurements}

All blood samples were collected from the cubital vein under fasting conditions ( $\geq 8 \mathrm{~h}$ since the last meal). Trained technicians measured blood pressure using a standard mercury sphygmomanometer, after the subjects had rested for at least $5 \mathrm{~min}$ in the sitting position. Waist circumference was measured at the point of the umbilicus level in the standing position. BMI $\left(\mathrm{kg} / \mathrm{m}^{2}\right)$ was calculated as weight divided by the square of the height in meters. High-density lipoprotein cholesterol (HDLC) and triglycerides were measured using conventional methods. Low-density lipoprotein cholesterol (LDL-C) was calculated using Friedewald's formula when the triglyceride level was $<4.5 \mathrm{mmol} / \mathrm{L}^{14}$

Each subject completed a self-administered questionnaire at baseline to assess medical history including current medications, smoking habits, and alcohol consumption. Hypertension was defined as a systolic blood pressure $\geq 140 \mathrm{mmHg}$, diastolic blood pressure $\geq 90 \mathrm{mmHg}$, or the use of antihypertensive agents. Diabetes was defined as a fasting blood glucose $\geq 7.0 \mathrm{mmol} / \mathrm{L}$, or the use of anti-diabetic agents. Dyslipidemia was defined as either LDL-C $\geq 3.6 \mathrm{mmol} / \mathrm{L}$, HDL-C $<1.0 \mathrm{mmol} / \mathrm{L}$, triglycerides $\geq 1.7 \mathrm{mmol} / \mathrm{L}$, or the use of anti-lipemic agents.

Until 1995, the underlying cause of death was determined based on death certificates coded according to the criteria of the International Classification of Diseases, ninth revision (ICD9). From 1995, the codes were translated into the corresponding ICD-10 codes. Deaths from IHD and stroke were defined as 410-414 and 430-438 (ICD-9); and I20-25 and I60-69 (ICD10), respectively. IHD and stroke were combined as CVD in the analyses.

\section{Statistical Analysis}

The mean duration of follow-up was 14.7 years, during which time there were 1,711 all-cause deaths, including 447 CVD deaths. The person-years studied were calculated as the period from baseline to either the first endpoint (death or emigration) or 31 December 2008.

Sex-specific hazard ratios and $95 \%$ confidence intervals (CIs) of all-cause and CVD deaths were calculated using Cox's regression models with data grouped according to waist circumference quintile by sex. The first quintile served as the reference. Model 1 was adjusted for age (continuous) and the study cohort using 2 dummy variables, whereas model 2 was adjusted further for smoking status (current smoker, ex-smoker, or never smoker) and alcohol consumption (regular drinker, ex-drinker, or never drinker). Model 3 was adjusted for the variables in model 2 plus hypertension, diabetes, and dyslipidemia (yes or no). The penalized spline (P-spline) method was used in the Cox regression models ${ }^{15}$ to examine non-linear dose-response relationships between waist circumference and the hazard ra- 


\begin{tabular}{|c|c|c|c|c|c|}
\hline \multicolumn{6}{|c|}{ WC quintile } \\
\hline & Q1 (low) & Q2 & Q3 & Q4 & Q5 (high) \\
\hline \multicolumn{6}{|l|}{ Men } \\
\hline $\mathrm{n}$ & 688 & 781 & 723 & 632 & 730 \\
\hline WC (cm) & $58.0-75.8$ & $76.0-81.0$ & $81.5-85.0$ & $85.5-89.5$ & $90.0-112.0$ \\
\hline Median WC (cm) & 72.0 & 79.0 & 84.0 & 87.0 & 93.0 \\
\hline Age (years) & $61.6 \pm 11.4$ & $58.8 \pm 11.1$ & $58.6 \pm 10.8$ & $59.0 \pm 10.3$ & $59.6 \pm 10.4$ \\
\hline BMI $\left(\mathrm{kg} / \mathrm{m}^{2}\right)$ & $19.5 \pm 1.7$ & $21.7 \pm 1.6$ & $23.1 \pm 1.5$ & $24.2 \pm 2.0$ & $26.4 \pm 3.0$ \\
\hline $\mathrm{SBP}(\mathrm{mmHg})$ & $126.4 \pm 21.1$ & $128.3 \pm 20.1$ & $130.9 \pm 19.0$ & $133.8 \pm 20.3$ & $136.2 \pm 20.0$ \\
\hline $\mathrm{DBP}(\mathrm{mmHg})$ & $74.9 \pm 10.9$ & $77.3 \pm 10.7$ & $79.8 \pm 10.1$ & $80.7 \pm 10.6$ & $83.2 \pm 11.3$ \\
\hline Hypertension (\%) & 29.9 & 34.3 & 34.0 & 42.4 & 51.4 \\
\hline Dyslipidemia (\%) & 33.7 & 46.5 & 57.5 & 64.9 & 66.0 \\
\hline Diabetes (\%) & 6.1 & 6.7 & 7.5 & 8.5 & 10.6 \\
\hline \multicolumn{6}{|l|}{ Smoking status (\%) } \\
\hline Current smoker & 58.5 & 51.4 & 42.2 & 45.1 & 40.9 \\
\hline Ex-smoker & 24.6 & 28.0 & 31.8 & 33.0 & 35.8 \\
\hline Never smoker & 16.9 & 20.6 & 26.1 & 22.0 & 23.3 \\
\hline \multicolumn{6}{|c|}{ Alcohol consumption (\%) } \\
\hline Regular drinker & 60.7 & 72.5 & 69.4 & 68.6 & 69.6 \\
\hline Ex-drinker & 5.5 & 4.8 & 5.0 & 5.6 & 4.4 \\
\hline Never drinker & 33.8 & 22.7 & 25.7 & 25.8 & 26.0 \\
\hline \multicolumn{6}{|l|}{ Women } \\
\hline $\mathrm{n}$ & 875 & 986 & 817 & 891 & 903 \\
\hline WC (cm) & $53.0-70.4$ & $71.0-76.0$ & $76.5-81.5$ & $82.0-87.5$ & $88.0-120.0$ \\
\hline Median WC (cm) & 67.0 & 74.0 & 79.0 & 84.0 & 92.0 \\
\hline Age (years) & $55.9 \pm 11.5$ & $57.4 \pm 10.8$ & $58.5 \pm 10.5$ & $59.7 \pm 10.2$ & $61.9 \pm 10.2$ \\
\hline $\mathrm{BMI}, \mathrm{kg} / \mathrm{m}^{2}$ & $20.0 \pm 2.0$ & $21.4 \pm 2.0$ & $22.7 \pm 2.1$ & $23.9 \pm 2.3$ & $26.3 \pm 2.9$ \\
\hline $\mathrm{SBP}(\mathrm{mmHg})$ & $122.7 \pm 20.5$ & $126.7 \pm 20.9$ & $129.5 \pm 20.9$ & $132.7 \pm 21.0$ & $136.4 \pm 20.5$ \\
\hline $\mathrm{DBP}(\mathrm{mmHg})$ & $73.6 \pm 10.7$ & $76.0 \pm 10.6$ & $77.6 \pm 11.3$ & $79.0 \pm 10.7$ & $81.0 \pm 10.7$ \\
\hline Hypertension (\%) & 23.5 & 29.7 & 34.3 & 40.7 & 51.2 \\
\hline Dyslipidemia (\%) & 35.0 & 47.1 & 53.0 & 64.3 & 70.0 \\
\hline Diabetes (\%) & 2.5 & 2.6 & 3.9 & 4.9 & 9.3 \\
\hline \multicolumn{6}{|l|}{ Smoking status, \% } \\
\hline Current smoker & 10.1 & 7.9 & 7.2 & 8.3 & 9.1 \\
\hline Ex-smoker & 2.3 & 1.8 & 2.5 & 2.8 & 4.0 \\
\hline Never smoker & 87.6 & 90.3 & 90.4 & 88.9 & 86.9 \\
\hline \multicolumn{6}{|c|}{ Alcohol consumption (\%) } \\
\hline Regular drinker & 22.5 & 20.4 & 19.8 & 20.7 & 16.6 \\
\hline Ex-drinker & 1.8 & 1.3 & 1.1 & 1.4 & 1.8 \\
\hline Never drinker & 75.7 & 78.2 & 79.1 & 77.9 & 81.6 \\
\hline
\end{tabular}

Data given as mean $\pm \mathrm{SD}, \mathrm{n}$ or $(\%)$.

Abbreviations as in Table 1.

tios for all-cause and CVD deaths. The degrees of freedom in the P-spline terms were selected automatically, based on Akaike's information criteria. The $\mathrm{P}$-spline function provided the linear and non-linear components in the model. The Pvalues for the non-linear trends were therefore determined by the significance of the non-linear P-spline term. Although the general spline curve may be influenced by a skewed distribution and the number of knots specified, these factors had little influence on the curves in our method, with these being more accurate up to the $99^{\text {th }}$ percentile of the distribution (ie, waist circumference of $103 \mathrm{~cm}$ in men and $104 \mathrm{~cm}$ in women). ${ }^{15} \mathrm{In}$ the Cox regression models, the linear trends were examined using continuous waist circumference data. The statistical tests were 2 -sided, with $\mathrm{P}<0.05$ being regarded as statistically significant. S-Plus version 8.1J (TIBCO Software, Palo Alto, CA,
USA), was used for statistical analysis.

\section{Results}

Table 1 lists the characteristics of each cohort in the study. Mean follow-up was 11.3 years in the Tanno and Sobetsu study, 14.2 years in the Suita study, and 17.9 years in the Hisayama study. There were 1,711 all-cause deaths during the follow-up period in the 3 cohorts combined. Mean waist circumference in the cohorts ranged from 79.0 to $81.7 \mathrm{~cm}$.

Table 2 lists the means and percentages of the cardiovascular risk factors at baseline, grouped according to sex-specific waist circumference quintile. Waist circumference was associated positively with systolic and diastolic blood pressure, hypertension, dyslipidemia, and diabetes. Men with a small waist 


\begin{tabular}{|c|c|c|c|c|c|c|c|}
\hline \multirow{3}{*}{ Men } & \multicolumn{5}{|c|}{ Quintile of WC } & \multicolumn{2}{|c|}{$P$ for trend } \\
\hline & Q1 (low) & Q2 & \multirow[t]{2}{*}{ Q3 } & Q4 & \multirow[t]{2}{*}{ Q5 (high) } & \multirow[t]{2}{*}{ Linear } & Non-linear \\
\hline & & & & & & & \\
\hline Person-years & 9,284 & 11,288 & 10,501 & 9,108 & 10,223 & & \\
\hline \multicolumn{8}{|l|}{ All-cause } \\
\hline No. deaths & 279 & 218 & 181 & 177 & 187 & & \\
\hline Model 1 & 1.00 (Ref.) & $0.75(0.63-0.90)$ & $0.68(0.56-0.82)$ & $0.79(0.66-0.96)$ & $0.72(0.59-0.86)$ & $<0.001$ & 0.087 \\
\hline Model 2 & 1.00 (Ref.) & $0.78(0.65-0.93)$ & $0.73(0.60-0.88)$ & $0.84(0.69-1.01)$ & $0.77(0.64-0.93)$ & 0.005 & 0.091 \\
\hline Model 3 & 1.00 (Ref.) & $0.76(0.63-0.91)$ & $0.72(0.59-0.87)$ & $0.81(0.66-0.98)$ & $0.73(0.60-0.89)$ & 0.001 & 0.11 \\
\hline \multicolumn{8}{|l|}{ CVD } \\
\hline No. deaths & 57 & 52 & 47 & 31 & 45 & & \\
\hline Model 1 & 1.00 (Ref.) & $0.91(0.62-1.33)$ & $0.91(0.61-1.34)$ & $0.71(0.46-1.10)$ & $0.90(0.60-1.33)$ & 0.29 & 0.12 \\
\hline Model 2 & 1.00 (Ref.) & $0.96(0.65-1.40)$ & $0.99(0.67-1.48)$ & $0.74(0.47-1.15)$ & $0.97(0.65-1.45)$ & 0.49 & 0.13 \\
\hline Model 3 & 1.00 (Ref.) & $0.92(0.63-1.36)$ & $0.95(0.64-1.42)$ & $0.67(0.43-1.06)$ & $0.87(0.57-1.32)$ & 0.22 & 0.16 \\
\hline \multicolumn{8}{|l|}{ Women } \\
\hline Person-years & 12,863 & 14,834 & 12,294 & 13,749 & 14,040 & & \\
\hline \multicolumn{8}{|l|}{ All-cause } \\
\hline No. deaths & 116 & 115 & 123 & 134 & 181 & & \\
\hline Model 1 & 1.00 (Ref.) & $0.76(0.59-0.98)$ & $0.86(0.67-1.12)$ & $0.75(0.59-0.97)$ & $0.83(0.65-1.05)$ & 0.29 & 0.029 \\
\hline Model 2 & 1.00 (Ref.) & $0.79(0.61-1.02)$ & $0.91(0.70-1.18)$ & $0.76(0.59-0.98)$ & $0.84(0.66-1.07)$ & 0.27 & 0.060 \\
\hline Model 3 & 1.00 (Ref.) & $0.81(0.62-1.05)$ & $0.94(0.73-1.22)$ & $0.80(0.62-1.03)$ & $0.86(0.67-1.10)$ & 0.36 & 0.16 \\
\hline \multicolumn{8}{|l|}{ CVD } \\
\hline No. deaths & 38 & 29 & 38 & 46 & 64 & & \\
\hline Model 1 & 1.00 (Ref.) & $0.61(0.37-0.98)$ & $0.83(0.53-1.30)$ & $0.81(0.53-1.25)$ & $0.91(0.61-1.36)$ & 0.73 & 0.29 \\
\hline Model 2 & 1.00 (Ref.) & $0.63(0.39-1.02)$ & $0.88(0.56-1.38)$ & $0.83(0.54-1.27)$ & $0.91(0.61-1.37)$ & 0.84 & 0.35 \\
\hline Model 3 & 1.00 (Ref.) & $0.64(0.39-1.03)$ & $0.90(0.57-1.43)$ & $0.85(0.54-1.32)$ & $0.90(0.59-1.38)$ & 0.90 & 0.43 \\
\hline
\end{tabular}

Non-linear trend $\mathrm{P}$ was computed using the $\mathrm{p}$-spline terms in the Cox regression models, with adjustment for the covariates in models 1,2 and 3 . Model 1 was adjusted for age and community. Model 2 was adjusted further for smoking and alcohol drinking. Model 3 was adjusted for the variables in model 2 plus hypertension, dyslipidemia, and diabetes.

$\mathrm{HR}$, hazard ratio. Other abbreviations as in Table 1.

circumference were more likely to be current smokers and less likely to be regular alcohol drinkers. The Pearson's correlation coefficient between waist circumference and BMI was 0.85 $(\mathrm{P}<0.001)$ in men and $0.74(\mathrm{P}<0.001)$ in women.

Table 3 lists the sex-specific hazard ratios for all-cause and CVD deaths, grouped according to the sex-specific quintile of waist circumference. We tested the association using 3 models adjusting for age and the study cohort (model 1); model 1 plus smoking status, and alcohol consumption (model 2); and the variables in model 2 plus hypertension, diabetes, and dyslipidemia (model 3). Non-linear trends were tested using the Pspline function, and P-spline curves drawn based on model 3 (Figure 1). In men, the hazard ratios for all-cause death were significantly lower in the second to fifth quintile range of waist circumference compared with the first quintile, and showed a significant inverse linear trend in all the models. The hazard ratio of the highest quintile of waist circumference in model 3 was 0.73 (95\% CI: 0.60-0.89). The inverse linear association between hazard ratio and waist circumference remained significant after adjustment for confounders in Model 3 ( $\mathrm{P}$ for trend $=0.001$, whereas the non-linear dose-response relationship between hazard ratio and waist circumference was not significant in either model (Figure 1A). For CVD deaths, although the fourth quintile of waist circumference had the lowest hazard ratio, the ratios changed very little with level of waist circumference in the 3 models, with non-significant linear and non-linear trends (Figure 1B).

In women, the hazard ratios for all-cause death in the sec- ond and fourth quintiles of waist circumference were significantly decreased in model 1 . The non-linear relationship in model 1 was also statistically significant ( $P$ for trend=0.029). In models 2 and 3, however, these associations were attenuated after further adjustment for conventional CVD risk factors (Figure 1C), with waist circumference showing no linear or non-linear association with CVD mortality risk (Figure 1D).

In addition, we analyzed all-cause mortality risk stratified by the presence or absence of hypertension, diabetes, or dyslipidemia in men (Table S1). Inverse linear associations were found in individuals with hypertension and individuals without dyslipidemia or diabetes, but we did not find any adverse effects of greater waist circumference regardless of these common diseases in the present analysis.

The multivariate-adjusted hazard ratios for all-cause and CVD mortality in men stratified by age $(<65$ years and $\geq 65$ years) are given in Table 4. Figure 2 shows the $\mathrm{P}$-spline fitting curves of the hazard ratios. The lowest hazard ratio for CVD was seen in the fourth quintile of waist circumference in men aged $<65$ years. Although there was no longer a linear association between waist circumference and CVD mortality, the non-linear association was significant ( $\mathrm{P}$ for non-linear trend $<0.001$ ) and the spline curve was U-shaped (Figure 2C). Using the lowest risk group (fourth quintile) as the reference group, the hazard ratio of the fifth quintile was increased significantly (hazard ratio $=2.45$; 95\% CI: $1.08-5.57$ ). In contrast, waist circumference had a linear association with decreased risk of all-cause mortality in men $\geq 65$ years. The spline curves in women are 

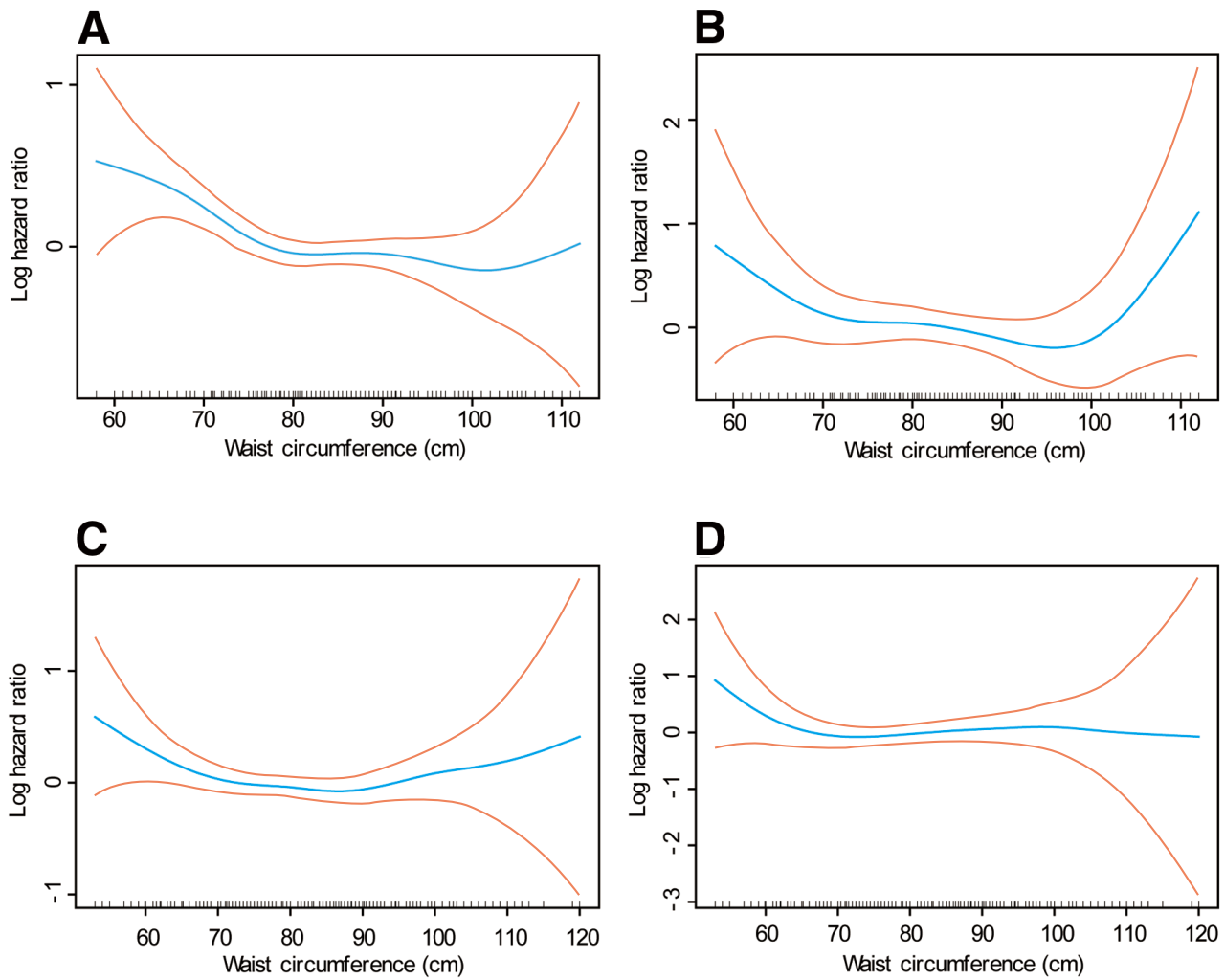

Figure 1. P-spline fitting curves (from Cox regression models) of hazard ratios (blue lines) and $95 \%$ confidence intervals (orange lines) for (A,C) all-cause and (B,D) cardiovascular disease (CVD) deaths vs. waist circumference in $(\mathbf{A}, \mathbf{B})$ men and $(\mathbf{C}, \mathbf{D})$ women. (A) Men, all-cause death (non-linear trend, $P=0.11)$; (B) men, CVD death ( $P=0.16)$; (C) women, all-cause death $(P=0.16)$; ( $D$ ) women, $C V D$ death $(P=0.43)$. The hazard ratios were adjusted for age, study community, smoking habits, alcohol consumption, hypertension, hyperlipidemia, and diabetes mellitus. Actual waist circumference used in the present analysis is marked on x-axis.

\begin{tabular}{|c|c|c|c|c|c|c|c|}
\hline \multirow[b]{3}{*}{$<65$ years } & \multicolumn{5}{|c|}{ Quintile of WC } & \multicolumn{2}{|c|}{$P$ for trend } \\
\hline & Q1 (low) & Q2 & Q3 & Q4 & Q5 (high) & Linear & Non-linear \\
\hline & & & & & & & \\
\hline Person-years & 6,013 & 8,267 & 7,570 & 6,756 & 7,496 & & \\
\hline \multicolumn{8}{|l|}{ All-cause } \\
\hline No. deaths & 82 & 81 & 76 & 72 & 72 & & \\
\hline $\mathrm{HR}$ & 1.00 (Ref.) & $0.86(0.63-1.17)$ & $0.90(0.65-1.24)$ & $0.86(0.62-1.19)$ & $0.82(0.59-1.15)$ & 0.36 & 0.089 \\
\hline \multicolumn{8}{|l|}{ CVD } \\
\hline No. deaths & 15 & 26 & 17 & 8 & 21 & & \\
\hline $\mathrm{HR}$ & 1.00 (Ref.) & $1.61(0.84-3.09)$ & $1.18(0.58-2.43)$ & $0.53(0.22-1.27)$ & $1.30(0.64-2.64)$ & 0.99 & $<0.001$ \\
\hline \multicolumn{8}{|l|}{$\geq 65$ years } \\
\hline Person-years & 3,271 & 3,021 & 2,932 & 2,352 & 2,727 & & \\
\hline \multicolumn{8}{|l|}{ All-cause } \\
\hline No. deaths & 197 & 137 & 105 & 105 & 115 & & \\
\hline $\mathrm{HR}$ & 1.00 (Ref.) & $0.72(0.57-0.90)$ & $0.63(0.49-0.80)$ & $0.81(0.63-1.03)$ & $0.71(0.55-0.90)$ & 0.003 & 0.11 \\
\hline \multicolumn{8}{|l|}{ CVD } \\
\hline No. deaths & 42 & 26 & 30 & 23 & 24 & & \\
\hline $\mathrm{HR}$ & 1.00 (Ref.) & $0.66(0.40-1.08)$ & $0.91(0.55-1.48)$ & $0.85(0.50-1.47)$ & $0.74(0.43-1.26)$ & 0.23 & 0.31 \\
\hline
\end{tabular}

The hazard ratios were adjusted for study cohort, age, smoking, alcohol drinking, hypertension, dyslipidemia, and diabetes. Non-linear trend $\mathrm{P}$ was computed using the $p$-spline terms in the Cox regression model adjusted for the same variables.

Abbreviations as in Tables 1,3 . 

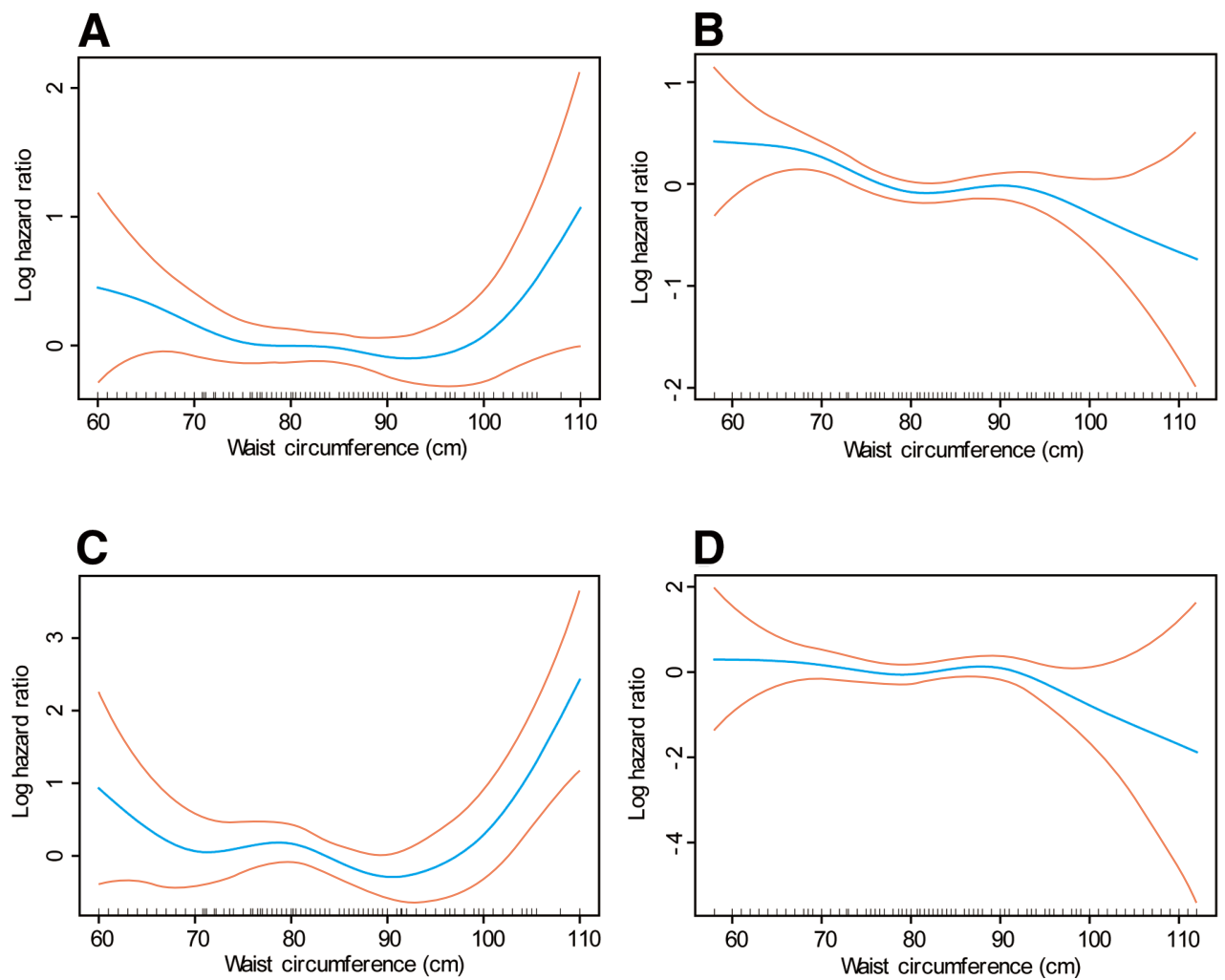

Figure 2. P-spline fitting curves (from Cox regression models) of hazard ratios (blue lines) and $95 \%$ confidence intervals (orange lines) for (A,B) all-cause and (C,D) cardiovascular disease (CVD) deaths vs. waist circumference in men $(\mathbf{A}, \mathbf{C})<65$ years and $(B, D) \geq 65$ years of age. (A) All-cause death, age $<65$ years (non-linear trend, $P=0.089$ ); (B) all-cause death, age $\geq 65$ years $(P=0.11)$; (C) CVD death, age $<65$ years $(P<0.001)$; (D) CVD death, age $\geq 65$ years $(P=0.31)$. The hazard ratios were adjusted for age, study community, smoking habits, alcohol consumption, hypertension, hyperlipidemia, and diabetes mellitus. Actual waist circumference used in the present analysis is marked on $\mathrm{x}$-axis.

shown in Figure $\mathbf{S 1 .}$

Figure 3 shows the dose-response curves between BMI and hazard ratios for all-cause and CVD deaths according to gender. In comparison with the curves for waist circumference in Figure 1, the non-linear trend was significant for all-cause and CVD deaths in women, but not in men. Hazard ratios for allcause and CVD deaths were increased moderately in obese women, although the $95 \%$ CIs became wider. When tested for linear trends, BMI levels were associated inversely with allcause mortality risk ( $\mathrm{P}$ for trend $<0.001$ ).

The analyses were repeated after excluding data from the first 5 years of follow-up (Table S2). We confirmed that all the associations were similar, with the non-linear association between waist circumference and CVD mortality in men becoming significant.

\section{Discussion}

This 14.7-year follow-up prospective study has shown that waist circumference is inversely associated with increased risk of all-cause death in men. We also showed that the non-linear dose-response relationship between waist circumference and CVD mortality risk is significant only in men aged $<65$ years. P-spline analysis, however, showed that the relationship between waist circumference and CVD mortality risk in middle- aged men was U-shaped, and that CVD mortality risk was increased only in the highest quintile of waist circumference $(\geq 90 \mathrm{~cm})$. In contrast, increased waist circumference did not influence all-cause or CVD mortality risk in elderly men, whereas a small waist circumference increased this risk.

Despite the close relationship between greater waist circumference and aggregation of CVD risk factors, ${ }^{16}$ abdominal obesity did not increase mortality risk. Although we examined this association using 3 regression models that included confounding factors such as smoking, alcohol drinking, and common diseases such as hypertension, the pattern of decreased risk for mortality did not change. Similar findings were seen in a previous Japanese study on the association between weight change and mortality risk. ${ }^{4}$ Considering the results of the present study as well as previous studies, the effect of obesity or weight gain on death appears to be considerably smaller in Japanese than Caucasian subjects. In contrast, recent large Japanese prospective studies demonstrated that overweight or obesity assessed using BMI raised the risk of incident stroke only in women, ${ }^{5}$ and that of incident coronary heart disease only in men. ${ }^{17} \mathrm{An}$ other meta-analysis in Japan also found that increased BMI was associated with a higher risk of incident stroke in both men and women, but a higher risk of coronary heart disease only in men. ${ }^{6}$ The results of these epidemiological studies suggest that obesity has a much different effect on CVD incidence than on 

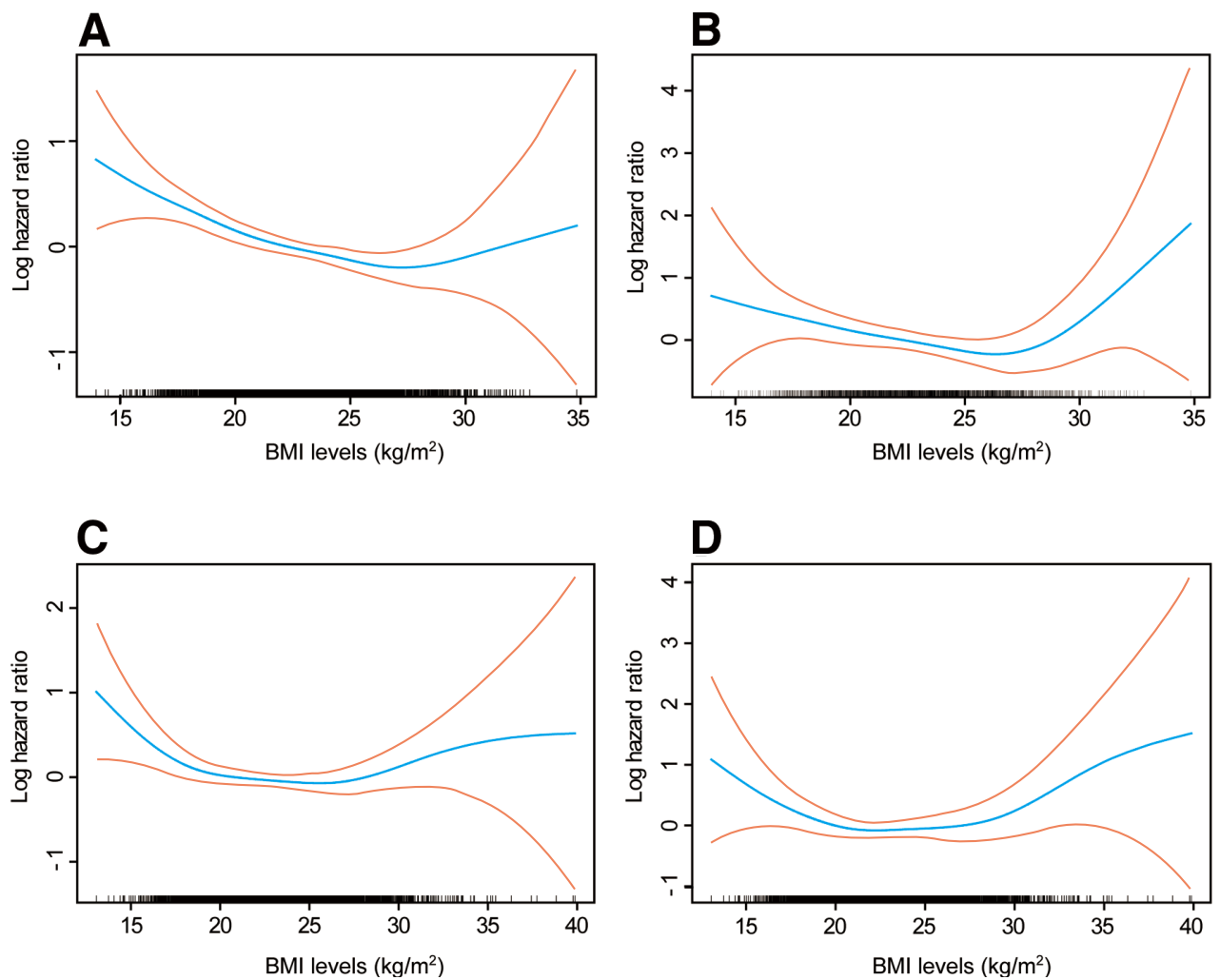

Figure 3. P-spline fitting curves (from Cox regression models) of hazard ratios (blue lines) and 95\% confidence intervals (orange lines) for (A,C) all-cause and (B,D) cardiovascular disease (CVD) deaths vs. body mass index (BMI) in $(\mathbf{A}, \mathbf{B})$ men and $(\mathbf{C}, \mathbf{D})$ women. (A) Men, all-cause death (non-linear trend $P=0.17$ ); (B) men, CVD death ( $P=0.079)$; (C) women, all-cause death $(P=0.026)$; (D) women, CVD death $(P=0.046)$. The hazard ratios were adjusted for age, study community, smoking habits, alcohol consumption, hypertension, hyperlipidemia, and diabetes mellitus. Actual BMl used in the present analysis is marked on the $\mathrm{x}$-axis.

CVD mortality.

Although it is not clear why the present findings are different from those in Western countries, this may be explained in part by the prevalence of obesity in the population. The prevalence of obesity (BMI $\geq 30 \mathrm{~kg} / \mathrm{m}^{2}$ ) in the present study was $1.2 \%$ in men and $2.3 \%$ in women, lower than the approximate $30 \%$ prevalence reported in US adults. ${ }^{18}$ Furthermore, based on vital statistics, the leading cause of death in Japan is cancer, being responsible for one-third of all deaths. The lower prevalence of obesity in Japan may reduce the effect of obesity on all-cause mortality risk. Furthermore, a previous Japanese prospective study showed that overweight subjects with metabolic syndrome did not have elevated CVD mortality compared with non-overweight subjects who had $\geq 2$ other conventional CVD risk factors. ${ }^{19,20}$

Waist circumference had a non-linear association with CVD mortality risk in middle-aged men, with this association being U-shaped. ${ }^{10}$ When compared with the fourth quintile, risk was increased at a waist circumference greater than approximately $90 \mathrm{~cm}$, which corresponded to the upper $20 \%$ of the entire distribution. In contrast, abdominal obesity was not associated with greater mortality risk in older men. Although this discrepancy is consistent with the findings of a previous US study, ${ }^{21}$ the explanation for the effect of aging on the relationship between obesity and mortality is not clear. We believe that older men who had a small waist circumference tended to lose weight due to nutrient deficiency, heavy smoking, or worsening diabetes. In this study, we found similar non-linear dose-response relationships between all-cause mortality risk and BMI, which correlated strongly with waist circumference. But we did not determine which parameter was better for predicting cardiovascular endpoints. As mentioned in the UK survey, a new study on this issue is required in Japanese adults. ${ }^{10}$

To the best of our knowledge, this is the first report to show an association between abdominal obesity and mortality risk in a large Japanese population-based study with long-term follow-up. The Tanno and Sobetsu study, ${ }^{12,22}$ the Suita study, ${ }^{23,24}$ and the Hisayama study ${ }^{25}$ are epidemiological studies that have been conducted for decades under high quality control, but several limitations should be noted. First, although we confirmed that the associations remained unaltered after excluding the first 5 years of follow-up data to eliminate the influence of latent disease at baseline, the results do not prove the existence of a cause and effect relationship. The increased risk of mortality was considered to be due to the consequence of ill-health related to weight loss and low body weight. ${ }^{26} \mathrm{Al}-$ though the present study demonstrated that the effect of increased waist circumference on mortality was comparable regardless of the presence or absence of hypertension, dyslipidemia, or diabetes, ill-health associated with low body weight was not fully controlled. Second, we did not assess the physical activity of the subjects, which may have confounded the associa- 
tion between obesity and mortality risk. ${ }^{27,28}$ Third, increased waist circumference may have a different effect on disease onset than on mortality risk, and the present study evaluated only mortality risk. Future studies on the effect of waist circumference on the incidence of CVD are therefore needed to address this issue. ${ }^{8}$

In conclusion, waist circumference was associated inversely with increased risk of all-cause death in men, but not in women. Although a non-linear statistical approach showed increased risk of CVD mortality with larger waist circumference in middle-aged men, a smaller waist circumference was generally associated with an increased risk of mortality in the general Japanese population. Similar findings have been reported in previous studies that measured BMI rather than waist circumference.

\section{Acknowledgments}

This study was supported by the Health and Labour Science Research Grants (H19-ShiShu-017, H20-SeiShu-013, and H21-SeiShu-015). We appreciate the work of all the staff in the Tanno and Sobetsu study, the Suita study, and the Hisayama study. We thank Dr. Yasunao Yoshimasa (principal investigator) and all members of the Suita City Health Center and the Suita Medical Association. We also thank all researchers and the staff of the Division of Preventive Cardiology for performing the medical examinations and study follow-up, and Satsuki-Junyukai, the volunteer who organized the Suita Study.

\section{Disclosures}

None.

\section{References}

1. Zheng W, McLerran DF, Rolland B, Zhang X, Inoue M, Matsuo K, et al. Association between body-mass index and risk of death in more than 1 million Asians. N Engl J Med 2011; 364: 719-729.

2. Tsugane S, Sasaki S, Tsubono Y. Under- and overweight impact on mortality among middle-aged Japanese men and women: A 10-y follow-up of JPHC study cohort I. Int J Obes Relat Metab Disord 2002; 26: $529-537$.

3. Cui R, Iso H, Toyoshima H, Date C, Yamamoto A, Kikuchi S, et al. Body mass index and mortality from cardiovascular disease among Japanese men and women: The JACC study. Stroke 2005; 36: $1377-$ 1382.

4. Saito I, Konishi M, Iso H, Inoue M, Tsugane S. Impact of weight change on specific-cause mortality among middle-aged Japanese individuals. J Epidemiol Community Health 2009; 63: 447-454.

5. Saito I, Iso H, Kokubo Y, Inoue M, Tsugane S. Body mass index, weight change and risk of stroke and stroke subtypes: The Japan Public Health Center-based prospective (JPHC) study. Int J Obes (Lond) 2011; 35: 283-291.

6. Yatsuya H, Toyoshima H, Yamagishi K, Tamakoshi K, Taguri M, Harada A, et al. Body mass index and risk of stroke and myocardial infarction in a relatively lean population: Meta-analysis of 16 Japanese cohorts using individual data. Circ Cardiovasc Qual Outcomes 2010; 3: $498-505$.

7. Yonemoto K, Doi Y, Hata J, Ninomiya T, Fukuhara M, Ikeda F, et al. Body mass index and stroke incidence in a Japanese community: The Hisayama study. Hypertens Res 2011; 34: 274-279.

8. Furukawa Y, Kokubo Y, Okamura T, Watanabe M, Higashiyama A, Ono Y, et al. The relationship between waist circumference and the risk of stroke and myocardial infarction in a Japanese urban cohort: The Suita study. Stroke 2010; 41: 550-553.

9. Pischon T, Boeing H, Hoffmann K, Bergmann M, Schulze MB, Overvad K, et al. General and abdominal adiposity and risk of death in Europe. N Engl J Med 2008; 359: 2105-2120.

10. Czernichow S, Kengne AP, Stamatakis E, Hamer M, Batty GD. Body mass index, waist circumference and waist-hip ratio: Which is the better discriminator of cardiovascular disease mortality risk?: Evidence from an individual-participant meta-analysis of 82864 participants from nine cohort studies. Obes Rev 2011; 12: 680-687.

11. de Hollander EL, Bemelmans WJ, Boshuizen HC, Friedrich N, Wallaschofski $\mathrm{H}$, Guallar-Castillon $\mathrm{P}$, et al. The association between waist circumference and risk of mortality considering body mass index in 65- to 74-year-olds: A meta-analysis of 29 cohorts involving more than 58000 elderly persons. Int J Epidemiol 2012; 41: 805-817.

12. Ohnishi H, Saitoh S, Takagi S, Katoh N, Chiba Y, Akasaka H, et al. Incidence of type 2 diabetes in individuals with central obesity in a rural Japanese population: The Tanno and Sobetsu study. Diabetes Care 2006; 29: 1128-1129.

13. Ohmura T, Ueda K, Kiyohara Y, Kato I, Iwamoto H, Nakayama K, et al. Prevalence of type 2 (non-insulin-dependent) diabetes mellitus and impaired glucose tolerance in the Japanese general population: The Hisayama Study. Diabetologia 1993; 36: 1198-1203.

14. Friedewald WT, Levy RI, Fredrickson DS. Estimation of the concentration of low-density lipoprotein cholesterol in plasma, without use of the preparative ultracentrifuge. Clin Chem 1972; 18: 499-502.

15. Govindarajulu US, Spiegelman D, Thurston SW, Ganguli B, Eisen EA. Comparing smoothing techniques in Cox models for exposure-response relationships. Stat Med 2007; 26: 3735-3752.

16. Choi SJ, Keam B, Park SH, Park HY. Appropriate waist circumference cut-offs to predict diabetes in the Korean population: The Korean Genome and Epidemiology Study. Circ J 2010; 74: 1357-1363.

17. Chei $\mathrm{CL}$, Iso $\mathrm{H}$, Yamagishi $\mathrm{K}$, Inoue $\mathrm{M}$, Tsugane $\mathrm{S}$. Body mass index and weight change since 20 years of age and risk of coronary heart disease among Japanese: The Japan Public Health Center-Based Study. Int J Obes (Lond) 2008; 32: 144-151.

18. Kuczmarski RJ, Flegal KM, Campbell SM, Johnson CL. Increasing prevalence of overweight among US adults. The National Health and Nutrition Examination Surveys, 1960 to 1991. JAMA 1994; 272: 205 211.

19. Saito I, Iso H, Kokubo Y, Inoue M, Tsugane S. Metabolic syndrome and all-cause and cardiovascular disease mortality: Japan Public Health Center-based Prospective (JPHC) Study. Circ J 2009; 73: 878-884.

20. Saito I. Epidemiological evidence of type 2 diabetes mellitus, metabolic syndrome, and cardiovascular disease in Japan. Circ J 2012; 76: $1066-1073$.

21. Kuk JL, Ardern CI. Influence of age on the association between various measures of obesity and all-cause mortality. J Am Geriatr Soc 2009; 57: 2077-2084.

22. Chiba Y, Saitoh S, Takagi S, Ohnishi H, Katoh N, Ohata J, et al. Relationship between visceral fat and cardiovascular disease risk factors: The Tanno and Sobetsu study. Hypertens Res 2007; 30: 229-236.

23. Kokubo Y, Kamide K, Okamura T, Watanabe M, Higashiyama A, Kawanishi K, et al. Impact of high-normal blood pressure on the risk of cardiovascular disease in a Japanese urban cohort: The Suita study. Hypertension 2008; 52: 652-659.

24. Kokubo Y, Nakamura S, Okamura T, Yoshimasa Y, Makino H, Watanabe M, et al. Relationship between blood pressure category and incidence of stroke and myocardial infarction in an urban Japanese population with and without chronic kidney disease: The Suita Study. Stroke 2009; 40: 2674-2679.

25. Kubo M, Hata J, Doi Y, Tanizaki Y, Iida M, Kiyohara Y. Secular trends in the incidence of and risk factors for ischemic stroke and its subtypes in Japanese population. Circulation 2008; 118: 2672-2678.

26. Wannamethee SG, Shaper AG, Walker M. Weight change, body weight and mortality: The impact of smoking and ill health. Int $J$ Epidemiol 2001; 30: 777-786.

27. Bellocco R, Jia C, Ye W, Lagerros YT. Effects of physical activity, body mass index, waist-to-hip ratio and waist circumference on total mortality risk in the Swedish National March Cohort. Eur J Epidemiol 2010; 25: 777-788.

28. Patel AV, Bernstein L, Deka A, Feigelson HS, Campbell PT, Gapstur $\mathrm{SM}$, et al. Leisure time spent sitting in relation to total mortality in a prospective cohort of US adults. Am J Epidemiol 2010; 172: 419429.

\section{Supplementary Files}

Supplementary File 1

Table S1. Multivariate-Adjusted HR for All-Cause Mortality in Men

Table S2. Multivariate HRs for All-Cause and CVD Mortality After Exclusion of Data From the First 5 Years of Follow-up

Figure S1. P-spline fitting curves (from Cox regression models) of hazard ratios and 95\% CIs for (A,B) all-cause and (C,D) CVD deaths, according to variation in waist circumference in women, stratified by age group.

Please find supplementary file(s);

http://dx.doi.org/10.1253/circj.CJ-11-1259 